\title{
Chyloptysis as a delayed sequela of penetrating thoracic trauma
}

Kyle G. Mitchell, MD, MSc, ${ }^{\mathrm{a}}$ Hope Feldman, MD, ${ }^{\mathrm{a}}$ Bryan F. Meyers, MD, MPH, ${ }^{\mathrm{b}}$ and

Mara B. Antonoff, MD, ${ }^{a}$ Houston, Tex, and St Louis, Mo

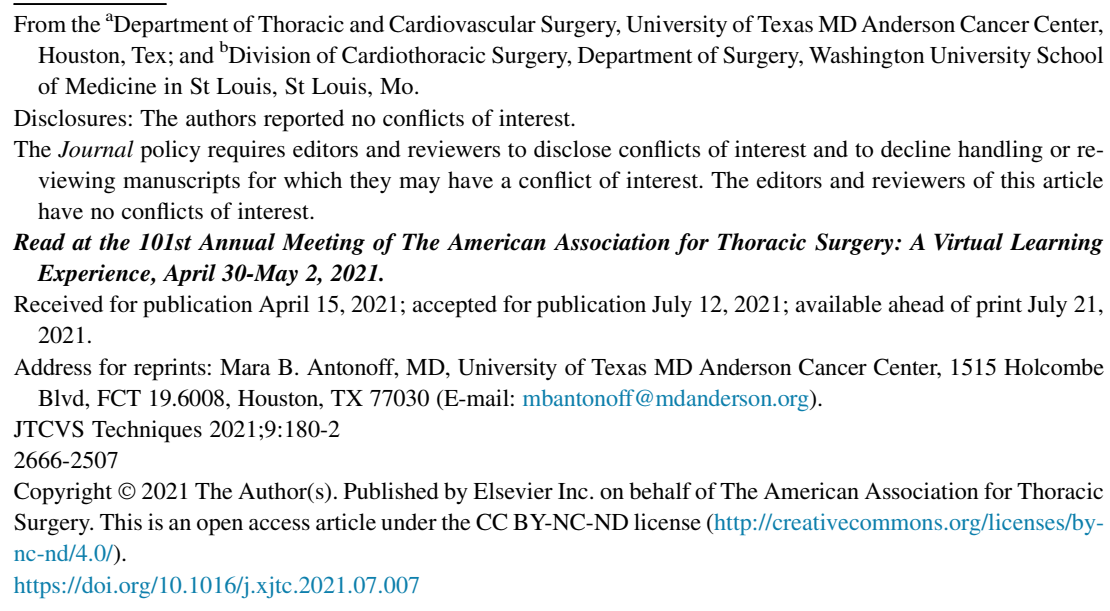

Video clip is available online.

We report the case of a young man who presented with a 3 -year history of progressive dyspnea, a productive cough, wheezing, and severe orthopnea with a sensation of drowning while lying supine. He reported thin, grayishwhite sputum (Figure 1, $A$ ) and expectoration of occasional bronchial casts (Figure 1, $B$ and $C$, Video 1).

His medical history was notable for asthma (controlled with inhaled bronchodilators and corticosteroids), gastroesophageal reflux disease, and penetrating injury to the left chest and left flank from stab wounds suffered more than a decade before presentation. Further detail regarding medical care received after his injury was unavailable; however, he denied having undergone operative intervention. He was a former smoker (15-pack-year history) and had quit 1 year before presentation. He had no known occupational exposures. Physical examination was unremarkable, with the exception of a healed right cervical scar and several healed scars on the left anterior chest.

Fiberoptic bronchoscopy had been performed by a pulmonologist at an outside institution before referral and demonstrated mucus impaction. Lymphangiography demonstrated normal lymphatic ducts in the abdomen and pelvis, a normal pattern of opacification of the thoracic duct, and an abnormal pattern of multiple tortuous, engorged

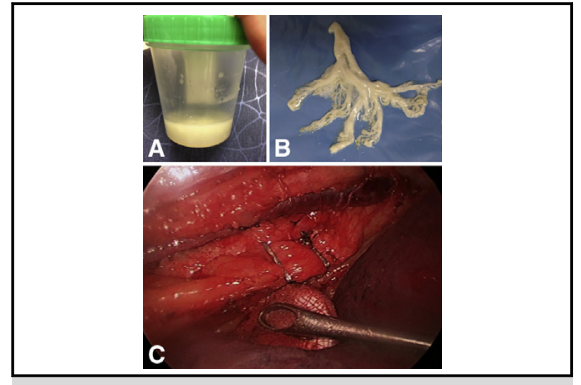

Milky sputum (A) and bronchial casts (B) after thoracic trauma; (C) thoracic duct ligation.

CENTRAL MESSAGE

We report a case of chyloptysis that presented more than 10 years following a penetrating thoracic injury. The patient was successfully managed with thoracoscopic thoracic duct ligation.

See Commentary on page 183 . lymphatic tributaries in the upper mediastinum, without obvious evidence of chyle leak (Figure 2, A). Subsequent computed tomography of the chest revealed collections of lipiodol within the mediastinum, hilum, and retroperitoneum with ground-glass opacities in the right lung consistent with a chemical pneumonitis (Figure 2, $B$ and $C$ ).

A low-fat diet was advised; however, the patient was noncompliant with dietary changes and desired definitive intervention. The patient was taken to the operating room for thoracic duct ligation via a right thoracoscopic approach. A retraction stitch was placed in the dome of the diaphragm to assist exposure and the posterior mediastinal pleura was opened (Figure 2,D). The thoracic duct was identified caudally within the right chest and dissected from surrounding structures (Figure 2, E). Following placement of cephalad clips and division of the duct, milky fluid was noted (Figure 2,F). The caudal aspect of the duct was clipped and the procedure concluded (Figure 2, G).

A regular diet without restrictions was reinstituted immediately postoperatively. His recovery was uneventful, and he was discharged home in good condition after having 


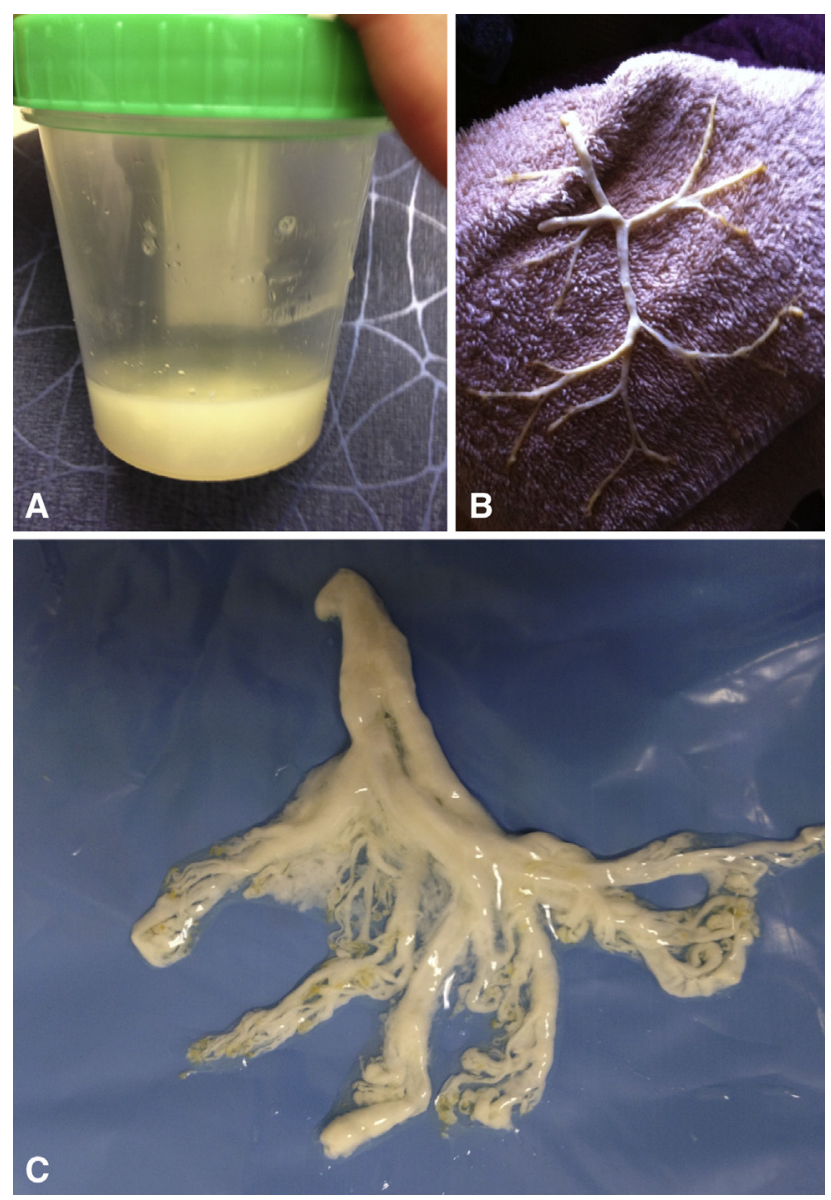

FIGURE 1. A man presented with a cough productive of milky sputum (A) and bronchial casts (B and C).

resumed a full-fat diet without any evidence of chyle leak. At follow-up evaluation 1 month postoperatively, the patient reported symptomatic improvement and reported no further expectoration of casts.

\section{DISCUSSION}

Chyloptysis is an uncommon finding that may be missed or ignored in the absence of chylothorax. It may arise from congenital or acquired (including idiopathic ${ }^{1}$ ) causes that result in (1) obstruction of the thoracic duct and/or its

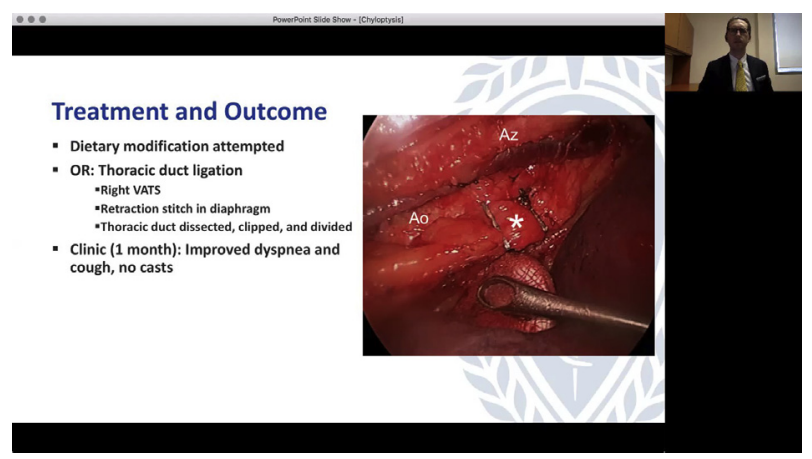

VIDEO 1. The authors present the case of a young man presenting with chyloptysis more than ten years after having suffered a penetrating thoracic injury. Video available at: https://www.jtcvs.org/article/S2666-2507(21) 00478-8/fulltext.

lymphatic tributaries, or (2) by broncholymphatic fistula. ${ }^{2-4}$ In this case, we speculate that the patient's index injury caused post-traumatic stenosis and obstruction of the upper mediastinal draining lymphatics, with subsequent lymphatic reflux and broncholymphatic fistula. A careful history and lymphangiography are crucial for diagnosis, and lymphangiographic examination is invaluable for the purposes of surgical planning. ${ }^{2,3}$ Analyses of expectorated sputum with Oil Red O stains and for the presence of triglycerides have been described as beneficial adjuncts. ${ }^{2-4}$ Dietary modification with adherence to a low-fat diet may be attempted. In the absence of alleviation of the underlying cause contributing to chyloptysis, ligation of the thoracic duct constitutes definitive surgical therapy. ${ }^{1,3}$ Alternatively, percutaneous thoracic duct embolization may in some cases successfully control chyloptysis and obviate the need for operative intervention.

Successful surgical management of chyloptysis following penetrating thoracic trauma has been previously described $^{5}$; however, in this previous report, the patient developed symptoms shortly after the injury. To our knowledge, this is the first reported case of chyloptysis as a delayed manifestation of remote penetrating thoracic injury. Vigilance is warranted when evaluating patients who have suffered any history of traumatic thoracic injury and present 


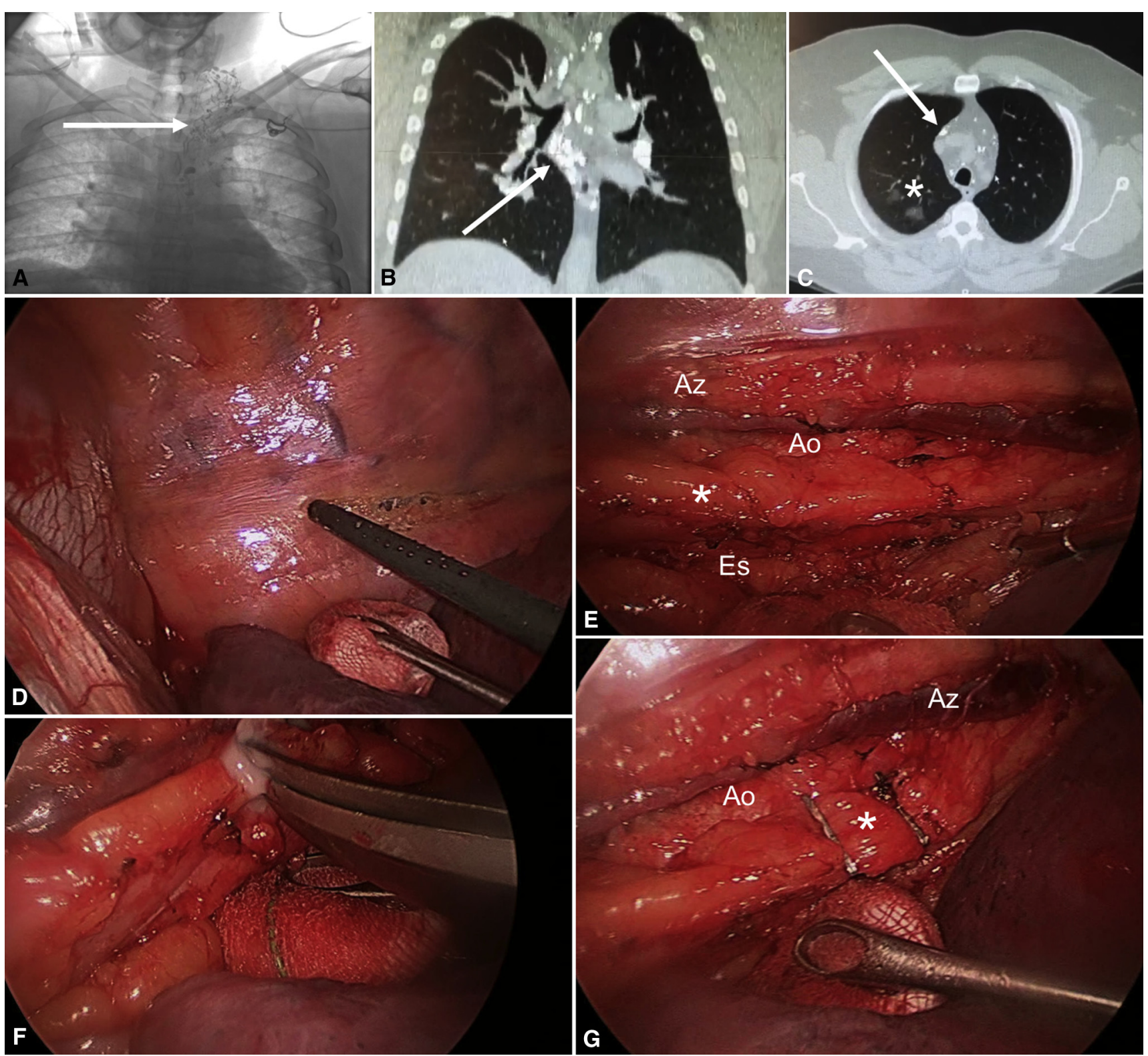

FIGURE 2. A, Lymphangiogram demonstrated tortuous lymphatic engorgement in the upper mediastinum without obvious evidence of chyle leak (arrow). $\mathrm{B}$ and $\mathrm{C}$, Computed tomography of the chest revealing collections of lipiodol within the mediastinum, hilum, and retroperitoneum (arrows) with ground glass opacities in the right lung (asterisk). D-G, intraoperative images demonstrating (D) incision in posterior mediastinal pleura after retraction of diaphragm using a retraction stitch, (E) identification of thoracic duct (asterisk), (F) milky fluid after division of thoracic duct, and (G) divided thoracic duct (asterisk) with visible nearby structures. The aorta (Ao), azygos vein (Az), and esophagus (Es) are indicated.

with a cough productive of bronchial casts or milky or cloudy white sputum so that proper therapeutic interventions may be promptly initiated.

\section{References}

1. Xu RB, Crouch G, Jurisevic C, Stuklis RG. Right video-assisted thoracoscopy thoracic duct ligation as treatment for a case of chyloptysis. J Thorac Cardiovasc Surg. 2013;145:e64-5.
2. Lim KG, Rosenow EC III, Staats B, Couture C, Morgenthaler TI. Chyloptysis in adults: presentation, recognition, and differential diagnosis. Chest. 2004;125: 336-40.

3. Gillaspie EA, Lim K, Nichols FC. Surgical management of chyloptysis. Ann Thorac Surg. 2018;105:e79-81.

4. Kato S, Umezawa H, Yano T, Ogasawara T, Kasamatsu N, Hashizume I. Chyloptysis after ligation of the thoracic duct. Intern Med. 2012;51:2403-6.

5. Yazicioglu A, Yazici U, Aydin E, Karaoglanoglu N. A strange bullet which caused chylomediastinum and chyloptysis. Thorac Cardiovasc Surg. 2014;62:372-4. 